\title{
Contribution of the Monkey Frontal Eye Field to Covert Visual Attention
}

\author{
Claire Wardak, ${ }^{1}$ Guilhem Ibos, ${ }^{1}$ Jean-René Duhamel, ${ }^{1}$ and Etienne Olivier ${ }^{1,2}$ \\ ${ }^{1}$ Institut des Sciences Cognitives, Unité Mixte de Recherche 5015, Centre National de la Recherche Scientifique-Université Claude Bernard Lyon I, 69675 \\ Bron, France, and ${ }^{2}$ Laboratoire de Neurophysiologie, Université Catholique de Louvain, B-1200 Brussels, Belgium
}

The frontal eye field (FEF) has long been regarded as a cortical area critically involved in the execution of voluntary saccadic eye movements. However, recent studies have suggested that the FEF may also play a role in orienting attention. To address this issue, we reversibly inactivated the FEF using multiple microinjections of muscimol, a $\mathrm{GABA}_{\mathrm{A}}$ agonist, in two macaque monkeys performing visually guided saccades to a single target. The effects of FEF inactivation were also studied in a covert visual search task that required monkeys to search for a target presented among several distractors without making any eye movements.

As expected, inactivating the FEF caused spatially selective deficits in executing visually guided saccades, but it also altered the ability to detect a visual target presented among distractors when no eye movements were permitted. These results allow us to conclude definitively to an involvement of the FEF in both oculomotor and attentional functions. Comparison of the present results with a similar experiment conducted in the lateral intraparietal cortex area revealed qualitatively different deficits, suggesting that the two areas may make distinct contributions to selective attention processes.

Key words: saccades; target selection; monkey; FEF; inactivation; visual salience

\section{Introduction}

The frontal eye field (FEF) is known to play a decisive role in the control of voluntary saccadic eye movements, as supported by converging evidence from single-unit, microstimulation, and lesion studies in nonhuman primates (Bruce and Goldberg, 1985; Bruce et al., 1985; Dias and Segraves, 1999). However, the FEF may also be involved in visual selection mechanisms and in the control of attention. For instance, visually responsive neurons in the FEF show early differential discharge activity to relevant and irrelevant stimuli presented in their response field during tasks requiring the selection of a saccade target among distractors (Schall and Hanes, 1993; Thompson et al., 1996). Another report shows that the activity of approximately half of the visual FEF neurons is modulated by covert attention during a nonoculomotor task in which the monkey responds manually to a stimulus appearing at cued versus noncued location (Kodaka et al., 1997), although another study provided conflicting results (Goldberg and Bushnell, 1981). In humans, functional imaging studies also suggest an implication of the FEF in target selection and/or attention control (Corbetta et al., 1998; Donner et al., 2000; Astafiev et al., 2003).

However, correlation is not causation, and to ascertain whether the FEF plays a functional role in attentional processing,

Received Aug. 9, 2005; revised Feb. 20, 2006; accepted Feb. 20, 2006

This work was supported by the French Ministry of Research (Action Concertée Incitative Neuroscience). We thank J.-L. Charieau for invaluable technical assistance.

Correspondence should be addressed to Jean-René Duhamel, Institut des Sciences Cognitives, Centre National de la Recherche Scientifique, 67 Bd Pinel, 69675 Bron, France. E-mail: duhamel@isc.cnrs.fr.

D01:10.1523/JNEUROSCI.3336-05.2006

Copyright $\odot 2006$ Society for Neuroscience $\quad 0270-6474 / 06 / 264228-08 \$ 15.00 / 0$ one must ask whether and how interference with FEF activity alters performance in appropriate, preferably nonsaccadic, tasks. For instance, microstimulation of the FEF below the threshold for eliciting saccades can selectively facilitate the detection of a visual stimulus located in the portion of space represented at the stimulation point (Moore and Fallah, 2001). Furthermore, visual responses in extrastriate visual area V4 are enhanced by FEF microstimulation, when the $\mathrm{V} 4$ receptive field location matches the region of the visual field represented at the FEF stimulation site (Moore and Amstrong, 2003).

These results suggest that the FEF possibly contributes to voluntary shifts of attention via control signals fed back to the visual cortex to enhance selectively the relative perceptual saliency of relevant objects. However, as acknowledged by the authors of the above experiments, one cannot eliminate the possibility that these microstimulation effects are not direct but are in fact mediated by other structures, such as the posterior parietal cortex, and in particular the lateral intraparietal area (LIP). Indeed, the area LIP is strongly connected to both the FEF and extrastriate visual cortex (Andersen et al., 1990; Blatt et al., 1990; Stanton et al., 1995; Bullier et al., 1996) and has been implicated in attentional mechanisms (Bisley and Goldberg, 2003; Wardak et al., 2004). To address this issue unequivocally, we inactivated the FEF using microinjections of muscimol, a $\mathrm{GABA}_{\mathrm{A}}$ agonist, while monkeys performed either visually guided saccades or a covert visual search task (see Fig. $1 A, B$ ).

\section{Materials and Methods}

General protocol. Two adult monkeys (Macaca mulatta monkey $\mathrm{M}$ and Macaca fascicularis monkey $\mathrm{G}$ ) weighing $\sim 6 \mathrm{~kg}$ were used in these experiments following procedures approved by the local animal care commit- 
tee, in compliance with the guidelines of European Community on Animal Care. Each monkey underwent a single surgical session under propofol anesthesia to prepare for chronic recording of eye movements and extracellular recording within the frontal cortex. The animals were implanted with scleral search coils (Judge et al., 1980) and a headrestraining device. A craniotomy was made over the left arcuate sulcus, and a stainless-steel recording chamber was implanted to allow access to FEF with microelectrodes and injection needles.

Throughout the duration of the experiments, the monkeys were seated in a primate chair with their head restrained, facing a tangent translucent screen $35 \mathrm{~cm}$ away, which spanned $\pm 55^{\circ}$ of the visual field. A mechanical lever, which could be displaced only vertically, was fixed on the chair at hand level in front of the monkey. The contact between the monkey and the lever and the press onto the lever were electrically detected. Behavioral paradigms, visual displays, and storage of both neuronal discharge and eye and hand movements were under the control of a personal computer running a real-time data acquisition system (REX) (Hays et al., 1982). Visual stimuli were back-projected onto the screen by a Davis (Drammen, Norway) DL-450 video projector. Eye movements were recorded with the magnetic search coil technique (Primelec, Zurich, Switzerland), and horizontal and vertical eye positions were digitized at 250 $\mathrm{Hz}$. All data analyses were performed off-line.

Behavioral tasks. Monkeys were trained to perform a visually guided saccade task and a covert visual search task. In the visually guided saccade paradigm, monkeys were required to maintain fixation for 1000-1600 ms until the fixation point disappeared. At this time, a visual target appeared at one of eight possible locations $\left(10^{\circ}\right.$ of eccentricity, radially distributed about the fixation point at $45^{\circ}$ intervals) in randomly interleaved order. The monkeys received a liquid reward if they made a saccade toward the target within $1000 \mathrm{~ms}$ of its appearance and maintained fixation there for at least $500 \mathrm{~ms}$.

The covert visual search task required the monkey to maintain fixation on a small spot of light at the center of the projection screen and search, while keeping their eyes on the fixation point, in the visual periphery for the presence of a predefined target in an array containing two, four, or eight items. A trial started when the monkey's hand was in contact with the lever and then the central fixation point appeared. From 300-1000 $\mathrm{ms}$ after the foveation of the fixation point, up to three visual search displays appeared in succession, each lasting $200 \mathrm{~ms}$, separated by a 1000 ms blank interval (see Fig. $1 B$ ). The monkeys had to press the lever within $900 \mathrm{~ms}$ after the appearance of a display, which contained the target. If no target was present, the monkeys refrained from responding and waited for the next display without breaking fixation. The target appeared in the first, second, or third display with equal probability, hence pressing the lever at random would result in $33.3 \%$ of correct answers. Trials were interrupted if the monkey pressed the lever when no target was present or failed to maintain fixation. Both monkeys used their right hand to answer.

Within a given trial, successive displays contained the same number of items, but the number of items per display varied randomly from one trial to the next. In most experiments, the visual items were circularly distributed at $10^{\circ}$ of eccentricity, half on the left side and half on the right side of the fixation point. However, for some control conditions tested in monkey $\mathrm{M}$, the visual items were aligned vertically on either side of the fixation point. The two lines were either at 3.5 or $7^{\circ}$ of the midline (tested in block), and the furthest item was located at $10^{\circ}$ of the fixation point. In a final control task performed by both monkeys, a single stimulus was presented at a random delay ranging between 300 and $1000 \mathrm{~ms}$ after foveation of the fixation point and at either $10^{\circ}$ right or $10^{\circ}$ left of the fixation point on the horizontal meridian. This stimulus was identical to the target used in the visual search task. The monkeys indicated its appearance by a manual response. This control was designed to test for a possible visual deficit induced by FEF inactivation, outside the context of visual search and in the absence of competition for attention.

In both the saccade and visual search tasks, ocular fixation had to be maintained in $2.5^{\circ}$ wide tolerance window, and in the saccade task, the eyes had to land in a $5^{\circ}$ wide window around the target.

Visual stimuli. In the saccade task, both the fixation point and the saccadic target consisted in a gray cross $\left(0.5^{\circ}\right)$. For the visual search task, the fixation point was also a gray cross. The target was a pink diamond shape. The basic visual search array tested in both monkeys was the detection of a conjunction target identified by a specific combination of two visual features, one shape and one color. The other combinations constituted the distractors (orange diamond, pink star, and orange star). All subtended the same visual angle of $1.8^{\circ}$. Two additional conditions were tested in which the target differed from the distractors by single visual feature. In the difficult feature search condition, the distractors were heterogeneous and consisted of three different shapes of the same color as the target (pink). In the easy feature search condition, there was only one distractor type of the same shape as, but different color from, the target (a blue diamond).

FEF localization. In one monkey (monkey M), the FEF was first located by using single-cell recordings. Single neuron activity was recorded extracellularly with tungsten microelectrodes (1-2 M $\Omega$ at $1 \mathrm{kHz}$; Frederick Haer, Bowdoinham, ME), which were lowered through stainless-steel guide tubes by means of a hydraulic microdrive (Narishige, Tokyo, Japan). Neuronal responses were recorded mainly in the anterior bank of the arcuate sulcus, while the monkey was performing a memory-guided saccade task. Visual and saccadic neuronal activities were recorded and used to identify FEF.

The localization of the FEF was confirmed in monkey M and determined in monkey $\mathrm{G}$ by using electrical microstimulation in anesthetized monkey. The monkeys underwent ketamine anesthesia (induction, 10 $\mathrm{mg} / \mathrm{kg}$, i.m.; maintenance, $3-4 \mathrm{mg} / \mathrm{kg} / \mathrm{h}$, i.v.) and were carefully monitored. Eye movements were monitored using a camera system coupled to an infrared source of illumination (Iscan, Burlington, MA). The stimulations were delivered by a stimulator (Neurolog) through tungsten microelectrodes (50-500 k $\Omega$ at $1 \mathrm{kHz}$; Frederick Haer). Stimulations consisted in trains of biphasic pulses (pulse duration, $0.25 \mathrm{~ms}$; train duration, $70 \mathrm{~ms}$; stimulation frequency, $300 \mathrm{~Hz}$ ) of varying intensity (range tested, 5-150 $\mu \mathrm{A}$ ). The FEF was defined as the cortical region, the stimulation of which elicited saccadic eye movements for an intensity $<50 \mu \mathrm{A}(\mathrm{Te}-$ hovnik and Sommer, 1997). We observed the known topographical organization of the FEF, along the arcuate sulcus, with very small saccades elicited in the most ventrolateral part and large saccades in the most dorsomedial part of the FEF (Bruce et al., 1985). This representation helped us to choose the injection points for muscimol experiments to cover the whole area FEF and to avoid diffusion of the muscimol in the neighboring areas. Injection tracks corresponded to sites evoking saccades at very low intensity $(10-40 \mu \mathrm{A})$.

FEF inactivation. A solution of 3-8 $\mu \mathrm{g} / \mu \mathrm{l}$ muscimol (Sigma, St. Louis, $\mathrm{MO}$ ) in saline was injected with a $5 \mu \mathrm{l}$ Hamilton syringe connected to a 29 gauge stainless-steel needle. Muscimol, a GABA $\mathrm{A}_{\mathrm{A}}$ agonist, was used because it interacts specifically with $\mathrm{GABA}_{\mathrm{A}}$ receptors and does not induce conduction block in fibers of passage. Three tracks were performed in each experiment and, along each track, one injection was made (see Fig. $1 A$ ). The volume injected at each site was $0.5 \mu \mathrm{l}$ and was delivered continuously in $7.5 \mathrm{~min}$ by an automatic pump system. The total amount of muscimol injected in each experiment ranged between 4.5 and $12 \mu \mathrm{g}$. Seven and eight injection experiments were made into the left frontal cortex of monkey $\mathrm{G}$ and monkey $\mathrm{M}$, respectively. When the injections were completed, we tested for the onset of muscimol effects with a saccadic task. This effect generally started 15-60 min after injection. The order of the different task conditions was counterbalanced across inactivation experiments, and control data were always obtained on the following day and in the same order of presentation. The entire duration of behavioral testing never lasted $>3 \mathrm{~h}$, well within the accepted temporal range of muscimol effects (Malpeli, 1999; Martin and Ghez, 1999). A physiological saline injection into FEF in monkey $\mathrm{M}$ served as an additional control for the specificity of the effects.

Data analysis. Preliminary data analysis did not indicate a systematic tendency for FEF inactivation to affect particular target locations within the contralesional hemifield. Thus, for the sake of presentation clarity, results for different target locations were grouped by hemifield. Intrinsic to the design of our visual search task, with its sequential presentation of up to three stimulus arrays, is the possibility that monkeys learned to anticipate the necessary presence of the target on the third array, after two arrays with no target in them. Surprisingly, no effect of the order of 
presentation within a trial was observed on reaction time. Third array targets were not responded to faster than first or second array targets, suggesting that the three stimulus arrays were processed in the same manner (two-way ANOVA, number of items times presentation order; monkey $\mathrm{G}$, presentation order factor, $p>0.40$, interaction, $p>0.20$; monkey $\mathrm{M}$, presentation order factor, $p>0.38$, interaction, $p>0.11$ ). Therefore, all subsequent statistical analyses were conducted on pooled data from the three types of trials. We used statistical procedures (ANOVA; Student-Newman-Keuls post hoc and $\chi^{2}$ tests) to evaluate the effects of muscimol inactivation on visual search behavior and on saccades directed contralaterally and ipsilaterally to the injection site.

Visually guided saccades were significantly disrupted, compared with the next day control session, in all individual inactivation experiments ( $n=6$ for monkey G; $n=8$ for monkey M). Significant effects on covert visual search performance were found in most experiments (four of six for monkey G; seven of eight for monkey M). Behavioral data obtained during a sham injection of saline solution in the FEF of monkey $\mathrm{M}$ showed no significant difference with control sessions. To increase the statistical power of the analyses presented below, we pooled the result from all experiments regardless of whether individual experiments yielded significant effects or not, and we compared these data with pooled data obtained on the day after each inactivation experiment.

\section{Results}

Effect of FEF inactivation on visually guided saccades

FEF inactivation led to spatially selective effects on saccade latencies (inactivation times saccade direction interaction, $p<0.0001$ in both monkeys). The latency of contraversive saccades increased by $>100 \mathrm{~ms}$ (monkey G, 365.7 vs $239.9 \mathrm{~ms}$ in control condition; monkey M, 290 vs $169.8 \mathrm{~ms}$ in control condition; both $p<0.05)$. In contrast, ipsiversive saccade latencies showed a small but significant decrease in latency (monkey G, 206.6 vs 231.5 ms; monkey M, 149.6 vs $162.3 \mathrm{~ms}$; both $p<0.05)$. FEF inactivation also reduced the frequency of contraversive saccades (percentage of executed saccades toward contralateral target during entire inactivation session versus control session, monkey $\mathrm{G}$, 53.9 vs $98.6 \%$; monkey $M, 60.8$ vs $99.6 \%$; both $p<0.0001$ ) (see Fig. $1 C$ for an example of a single injection session resulting to a strong effect on saccade frequency) and amplitude (monkey G, 6.81 vs $9.95^{\circ}$; monkey $\mathrm{M}, 6.29$ vs $9.05^{\circ}$; both $p<0.05$ ), whereas ipsiversive saccadic frequency and amplitude were unaltered. These results are consistent with a direct implication of the FEF in the control of visually guided saccades, as reported in previous studies (Sommer and Tehovnik, 1997; Dias and Segraves, 1999) and confirm the accurate location of our injections into the FEF.

Effect of FEF inactivation on conjunction search performance We first report the behavioral effect of FEF inactivation on covert visual search using conjunction stimuli, which are generated by a combination of one of two colors and one of two shapes. The target corresponded to one of the four possible combinations, the three remaining ones being used to produce distractors. The time necessary to find the target in this type of task is an approximately linear function of the number of items in the display, commonly taken as evidence of a serial search process involving multiple attentional shifts. In the control condition, we indeed obtained positive search slopes (monkey G, $7.32 \mathrm{~ms} /$ item; monkey M, 7.85 ms/item).

Statistical analyses (ANOVA and Student-Newman-Keuls post hoc tests) were conducted on pooled inactivation and control data to assess inactivation effects on visual search performance as a function of target location and number of items in the display. FEF inactivation produced significantly longer reaction times for contralesional targets (Fig. 2A) (monkey G, 379.2 vs $352.7 \mathrm{~ms}$ in control, inactivation main effect $p=0.0013$; monkey $\mathrm{M}, 386$ vs
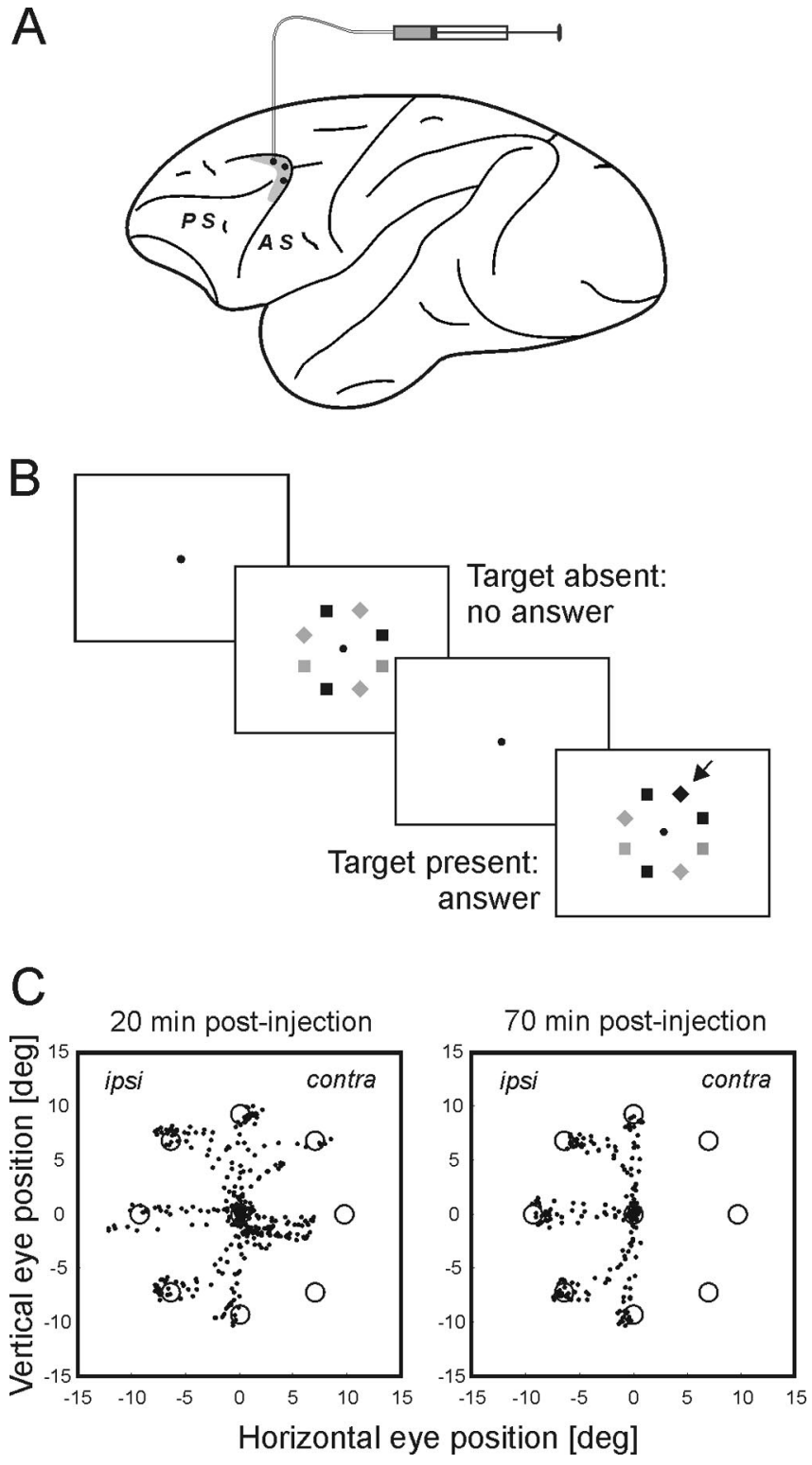

Figure 1. Covert search task procedure. $A$, Schematic representation of the left hemisphere of the monkey brain. The gray area represents the extent of the FEF established by microstimulation. The black dots correspond to the three median positions where muscimol was injected during one experiment. PS, Principal sulcus; AS, arcuate sulcus. $\boldsymbol{B}$, Time course of the visual search task. While the monkey fixates centrally, up to three successive search displays are presented in succession. The monkey has to depress a lever when the target is present among the objects. The target is represented here as the dark diamond. C, Examples of saccadic deficits induced by FEF inactivation. The monkey performed visually guided eye movements toward one of eight possible locations (same eccentricity than the objects in the visual search task). The plots show saccade traces, sampled at $250 \mathrm{~Hz}$, recorded at the beginning of the muscimol effect (20 min after injection), and later (70 min after injection) during a session in which contraversive saccades were virtually abolished at the peak of inactivation effect. The contralesional side (contra) of space is depicted on the right. Open circles represent the positions of the fixation point and of the target locations. ipsi, Ipsilesional side.

$358.5 \mathrm{~ms}$ in control, inactivation main effect $p<0.0001$ ). In both monkeys, there was a significant overall effect of the number of items $(p<0.005)$. Although there was a significant interaction between inactivation and number of items in one animal (monkey $\mathrm{M}, p<0.05$ ), the search time increase was not proportional to the number of items, and there was no change in the visual search slope. Detection time for ipsilesional targets was unaffected by FEF inactivation in monkey $\mathrm{G}$ (inactivation main effect, $p>0.48$; 
A Contralesional target

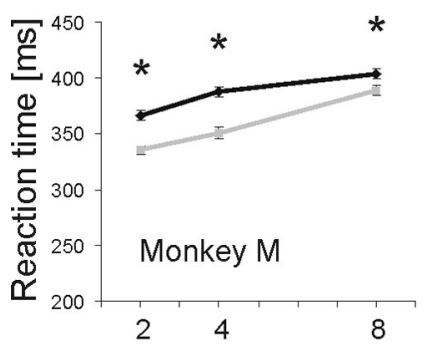

\section{B Ipsilesional target}

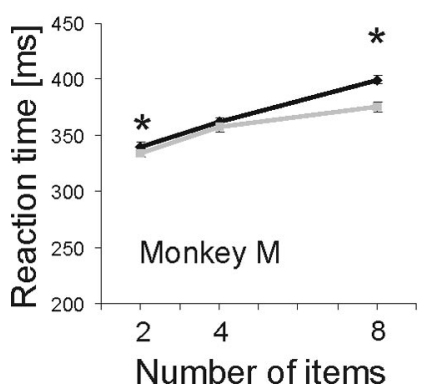

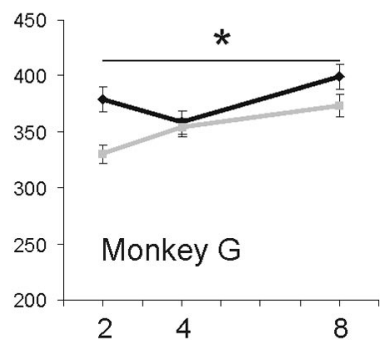

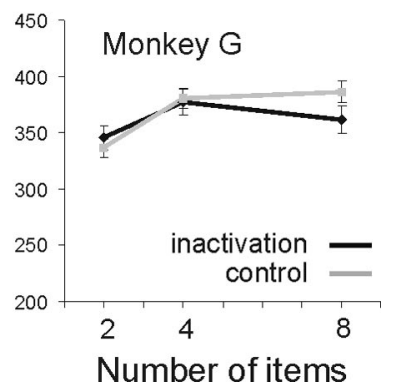

Figure 2. Effect of FEF inactivation on search time for a conjunction target. Detection time ( $m s \pm S E M$ ) is presented as a function of the number of items in the display, when the target is contralateral $(\boldsymbol{A})$ and ipsilateral ( $\boldsymbol{B})$ during the control (gray line) and the inactivation condition (black line). Results for monkey $M$ are displayed on the left; results for monkey $G$ are on the right. ${ }^{*} p<0.05$.

inactivation times number of item interaction, $p>0.27)$ but showed a moderate increase in monkey M (Fig. $2 B$ ) (inactivation times number of item interaction, $p=0.029$; significant post hoc tests at two and eight items, $p<0.05$ ).

\section{Effect of FEF inactivation on feature search performance}

Search for a conjunction target is a form of serial or effortful search, which necessitates, at some processing stage, the perceptual binding of the defining features of the searched stimulus (e.g., color and shape). Other kinds of search display can preserve this serial character (i.e., produce a positive search slope) but without involving any feature binding requirements. With yet other types of displays, detecting the target appears to be effortless (i.e., the search slope is flat), as though all items within the display were processed in parallel rather than serially. To determine whether visual search impairments consequent to FEF inactivation are attributable to inefficient feature binding and to establish whether the deficits are limited to serial search tasks, we introduced two additional target/distractor configurations.

In single feature search, distractors differ from the target by only one feature, hence no binding is involved, but the nature of the distractors used determines whether the task will be "serial" or "parallel". We tested the effect of FEF inactivation on an easy (parallel) feature search task involving a difference in color between the target and distractors and a homogeneous set of distractors (control search slopes for monkey G, $1.34 \mathrm{~ms} / \mathrm{item}$; for monkey M, $0.33 \mathrm{~ms} /$ item). We also used a difficult (serial) feature search condition involving a difference in shape between the target and distractors and a heterogeneous set of distractors. The difficulty level, as inferred from the search slopes (monkey G, $8.32 \mathrm{~ms} /$ item; monkey M, $10 \mathrm{~ms} /$ item), closely matched that of the conjunction condition.
A Difficult feature search

Contralesional target
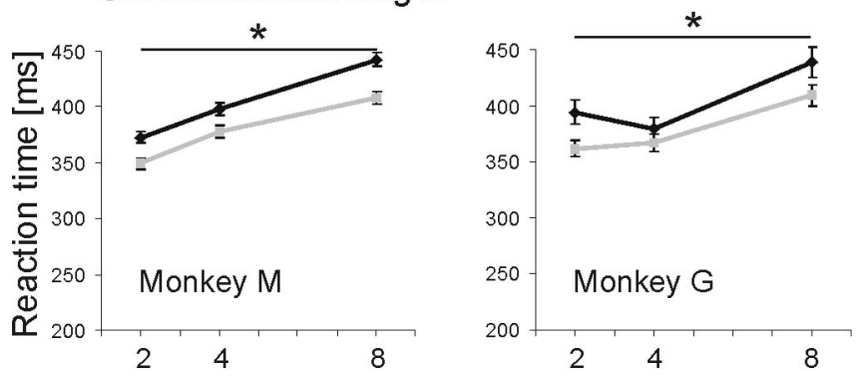

Ipsilesional target
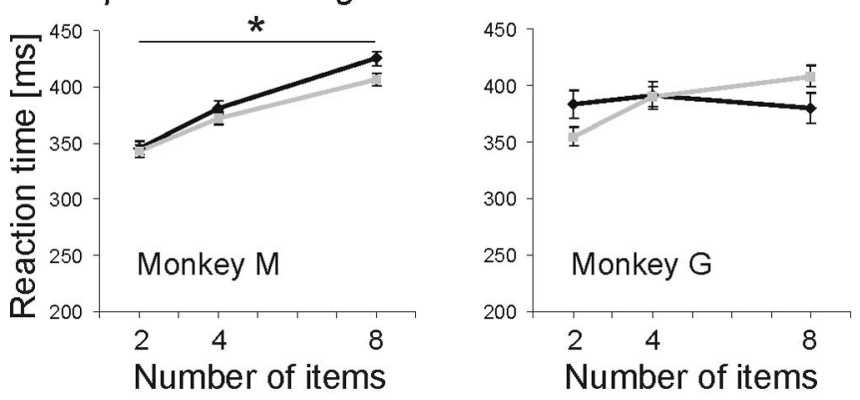

\section{B Easy feature search}

\section{Contralesional target}
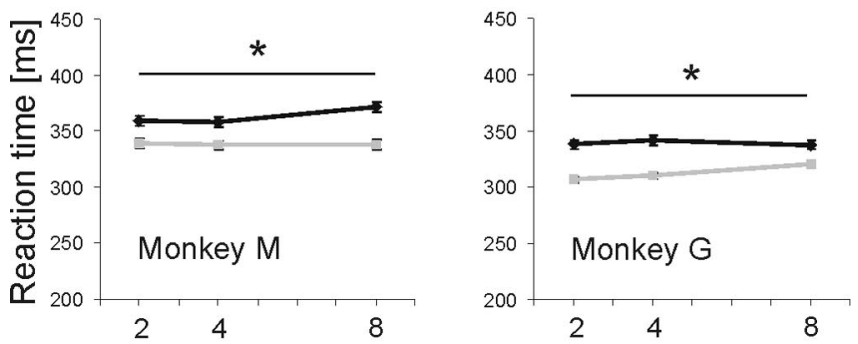

Ipsilesional target
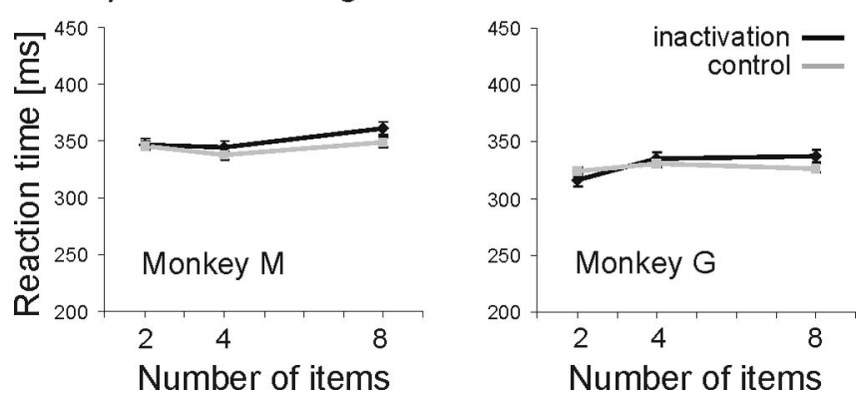

Figure 3. Effect of FEF inactivation on search time during a difficult, low-saliency $(\boldsymbol{A})$ and easy, high-saliency $(\boldsymbol{B})$ feature search task. Results for a contralesional target are presented at the top of each panel, and those for an ipsilesional target are presented at the bottom. Results for monkey $M$ are displayed on the left, and results for monkey $G$ are on the right. Conventions are the same as those in Figure 1.

Inactivating FEF increased search time for contralesional targets in the difficult feature search condition (Fig. $3 A$ ) (inactivation main effect, $p<0.003$ for both monkeys). As in the conjunction condition, detection of ipsilesional targets was unaltered (monkey G) or moderately impaired (monkey $\mathrm{M}$, inactivation main effect $p=0.03$; mean reaction time increase of $10.3 \mathrm{~ms}$ against $25.8 \mathrm{~ms}$ for the contralesional target, mostly because of 

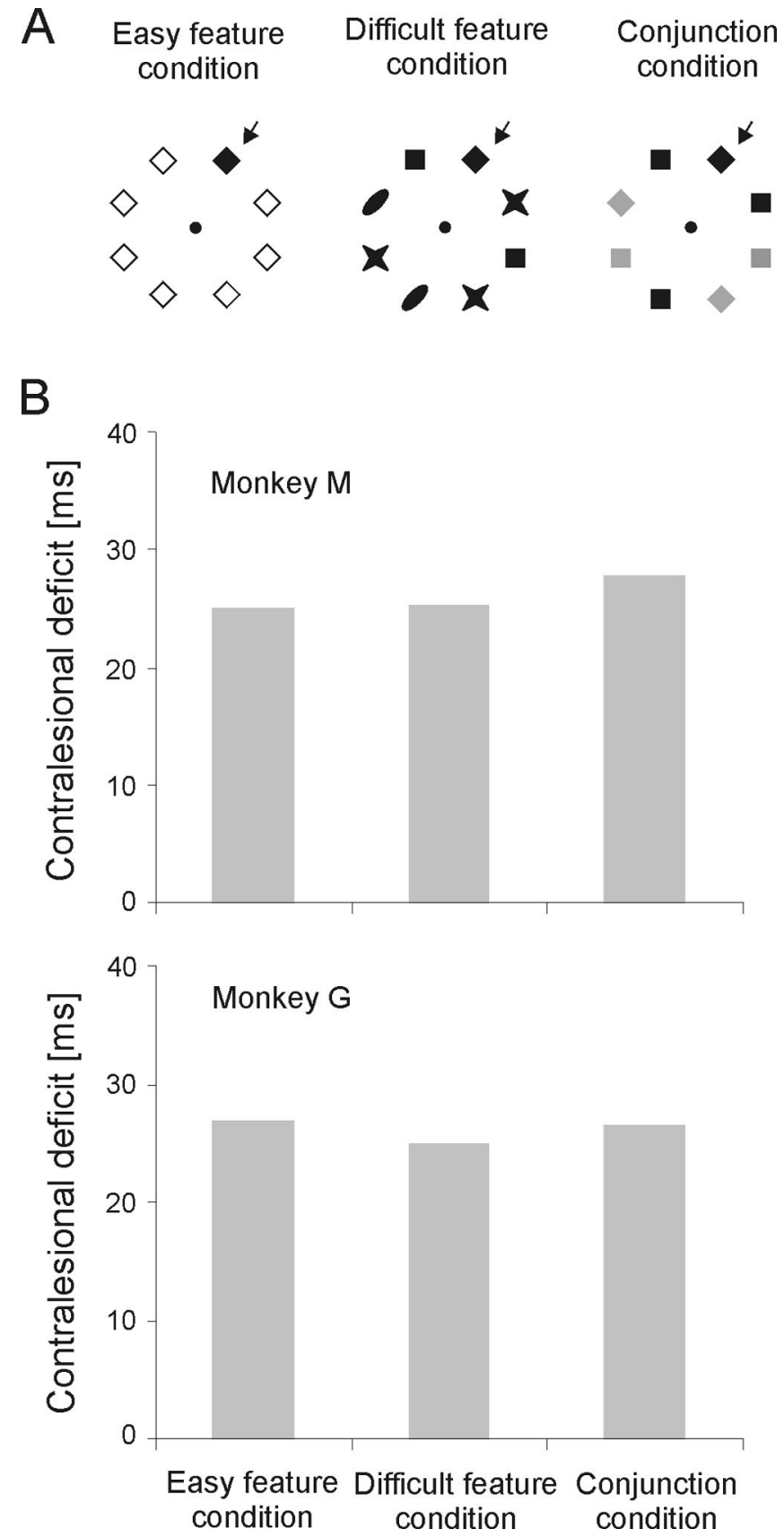

Figure 4. Summary of the deficits induced by the FEF inactivation. $A$, Example of eight-item displays used in the different visual search conditions. Actual colors were different. The target is indicated by the arrow. $\boldsymbol{B}$, The contralesional deficit (ms) for each monkey is shown, measured as the mean of the increase of reaction time for detecting a contralesional target in two-, fourand eight-item conditions compared with the control conditions.

the eight-item condition). We were surprised to find that the monkey's performance for contralesional targets were altered in the easy feature search condition to the same extent as in the difficult search condition (Fig. 3B) (inactivation main effect, $p<$ 0.0001 for both monkeys). Ipsilesional performances were unaffected.

To summarize, we found that FEF inactivation impaired visual search, regardless of the task difficulty or feature binding requirement: for both monkeys and in all three visual search conditions, search time for contralesional targets increased by 25-28 ms (Fig. 4). These effects cannot be simply explained by a general increase in reaction times to contralesional visual stimuli, which could unveil a more elementary visual deficit. Indeed, to test for this possibility, we used a task in which the monkeys had to detect the unpredictable appearance of the target item presented alone, either on the left or on the right side of space. In this condition, manual reaction times were never found longer after FEF inactivation than in the control condition (monkey $\mathrm{M}$, $p>0.8$; monkey G, $p>0.15$ ).

\section{Origin of the ipsilesional deficit in monkey $M$}

Because the visual representation in the FEF is mainly contralateral, we were surprised to find ipsilesional impairments after inactivating this area. Although this was observed in only one monkey, its level of reproducibility (the effect was significant or nearly significant in 13/19 individual data samples) and the fact that we never observed such effects after LIP inactivation in the same animal (Wardak et al., 2004) led us to perform additional tests to uncover the origin of this deficit. The magnitude and consistency of this effect was greatest in the eight-item configuration. Although all objects were at the same radial eccentricity, the spatial arrangement of stimuli in this configuration differed from the two- and four-item ones in that some objects were closer to the vertical midline than others (Fig. $5 A$ ).

This difference led us to consider two possible explanations for the ipsilesional deficits. A first possibility is that, because FEF neurons have receptive fields straddling the vertical meridian, the deficit produced by a unilateral injection might encroach into the portion of the ipsilesional field near the midline. The fact that we have seen it in only one animal might correspond to a difference between the injection site locations in the two monkeys. Alternatively, in a serial search task with stimuli placed at various distances from the midline, attention shifts may occur in different directions within the same hemifield (e.g., both left and right shifts within the right hemifield). Assuming that FEF controls all contraversive shifts of attention, and not only shifts toward the contralateral hemifield, its inactivation could slow down shifting attention from an object far from the midline to one close to the midline. According to this account, the difference observed between the two monkeys could reflect different attentional strategies. Supporting this conjecture, we found that in the control condition, monkey $\mathrm{M}$ systematically detected more rapidly targets placed far from the midline than those placed close to the midline $(p<0.02)$, suggesting that this monkey's attention was preferentially directed to the most peripheral objects. In contrast, monkey $\mathrm{G}$ seemed to detect targets just as quickly for all locations $(p>0.4)$, suggesting a more homogeneous distribution of attention.

To distinguish between these two hypotheses, we performed an additional experiment in monkey $\mathrm{M}$. All items were distributed in two columns placed either far from the midline $\left(7^{\circ}\right.$; mimicking the furthest locations in the standard eight-item display) (Fig. $5 B$, top) or near the midline $\left(3.5^{\circ}\right.$; mimicking the nearest positions) (Fig. $5 C$, top). Note that the spatial hypothesis predicts a deficit in the near but not in the far configuration, whereas the directional hypotheses predicts no deficit in any of these configurations. The results were consistent with latter hypothesis: after FEF inactivation, search time for ipsilateral targets did not differ from control performance in either of the two display configurations (Fig. $5 B, C$, bottom) (close, effect of the inactivation $p>$ 0.35 , interaction $p>0.8$; far, inactivation $p>0.45$, interaction $p>0.2$ ), whereas search for contralesional targets remained impaired, and the ipsilesional deficit was still present in the standard 


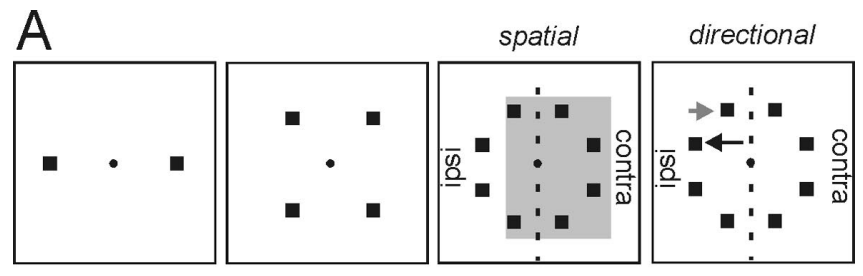

B

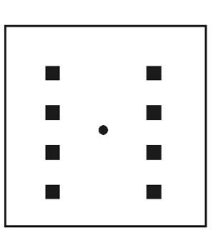

Contralesional target

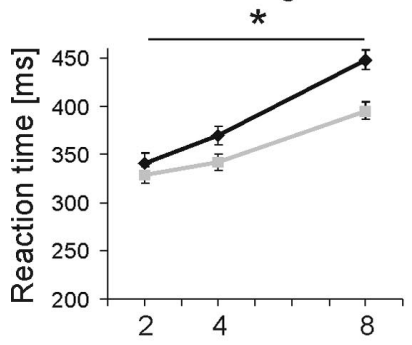

Ipsilesional target

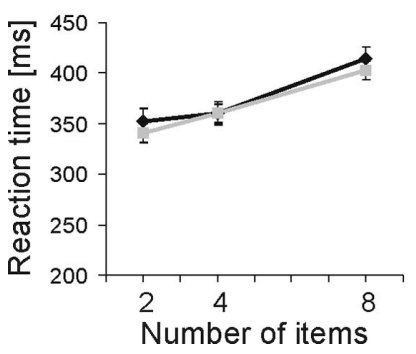

Figure 5. Hypotheses and controls to explain the presence of ipsilesional deficits. $\boldsymbol{A}$, Standard spatial configuration of the items for the two-, four-, and eight-item conditions. Two contrasting hypotheses to explain the deficit in the eight-item condition are illustrated: according to a spatial hypothesis, the region of visual space affected by FEF inactivation (gray area) could include a portion of the ipsilateral field; according to the directional hypothesis, the speed or probability of contraversive shifts of attention (gray arrow) are diminished compared with ipsiversive shifts (black arrow; gray area). B, C, FEF inactivation effect on the detection of a contralesional target (top part) and an ipsilateral target (bottom part) when all items are vertically aligned at 7 and at $3.5^{\circ}$ of eccentricity, respectively. Conventions are the same as those in Figure 1.

circular array condition (378.1 vs $361.8 \mathrm{~ms}$ in the eight-item configuration for inactivation and control, respectively; $p<0.002$ ).

\section{Effect of FEF inactivation on errors}

Because each display lasted for a relatively long time (200 ms) and was not followed by a mask, our search task might have favored accuracy over speed, hence possibly diminishing the sensitivity of detection errors as an index of performance. Nevertheless, we found that FEF inactivation produced a general increase in error rate in all search array configurations (monkey $\mathrm{M}, 30$ vs $12.1 \%$ for conjunction, 37 vs $15 \%$ for difficult feature, 32.2 vs $7 \%$ for easy feature search, $\chi^{2}, p<0.0001$; monkey G, 50.6 vs $17.6 \%$ for conjunction, 53.8 vs $30.1 \%$ for difficult feature, 33.1 vs $3.9 \%$ for easy feature search, $\chi^{2}, p<0.0001$ ).

We separated trials in which the monkey maintained central fixation but failed to detect the target (misses) or responded when no target was present (false alarms) and trials interrupted by failure to suppress eye movements (saccade). Similar effects of FEF inactivation were observed in the two serial search conditions (conjunction and difficult feature search) (Fig. 6): the false alarm rate increased significantly $\left(\chi^{2} ; p<0.02\right.$ for monkey $\mathrm{M}$; $p<0.0001$ for monkey $\mathrm{G}$, for each condition), but miss rates, which were generally low, changed only modestly either toward an increase in monkey $\mathrm{M}\left(\chi^{2}, p<0.04\right)$ or toward a decrease in monkey $\mathrm{G}\left(\chi^{2}, p<0.05\right)$. Both monkeys made significantly larger numbers of saccades, and these were mainly directed toward an ipsilesional object (for both monkeys and both conditions; $\chi^{2}, p<0.0001$ for ipsiversive, $p>0.05$ for contraversive saccades). For the easy feature search, the main significant effect of FEF inactivation was a large increase of ipsiversive saccade errors $\left(\chi^{2}, p<0.0001\right.$ for both monkeys; also a small increase of contraversive saccades in monkey $\mathrm{M}, \chi^{2}, p<0.001$; and a small increase of false alarms in monkey $\mathrm{G}, \chi^{2}, p<0.0001$ ).

\section{Discussion}

The major finding of the present study is that the FEF has an indisputable functional role in covert attentional processes, in addition to saccadic behavior. The deficits observed during FEF inactivation in the visual search task are neither motor nor purely visual in nature. Indeed, given that the required behavioral response was held constant, the fact that detection time increased in a spatially selective manner excludes any possible contribution of motor factors to the reported inactivation effects. The control detection task also shows that the monkeys were able to detect correctly and rapidly a contralesional visual stimulus when not in competition with others objects. This is in agreement with the observation that monkeys do not exhibit changes in contrast sensitivity after a permanent lesion of the FEF (Schiller and Chou, 2000), ruling out purely sensorial deficits. Detection performance deficits after FEF lesions thus emerge only in conditions in which visual attention and selection are required.

Previous studies have shown that visually responsive neurons in the FEF exhibit differential activity to targets and distractors during search tasks (Schall and Hanes, 1993; Thompson et al., 1996; Bichot and Schall, 1999). Our results provide strong evidence that these neurophysiological phenomena reflect more than a correlation between neuronal activity and behavior. Because the disruption of FEF neuronal activity impairs covert visual search performance, such FEF neuronal activity must clearly be functionally important for attentional processing. Experiments that used electrical microstimulation to interfere with brain function also provide evidence of a FEF contribution to selective attention (Moore and Amstrong, 2003; Moore and Fallah, 2004). However, a question that remained open until now is the causal chain of the observed effects. The present study addresses this issue, because the consequence of inactivating FEF can be compared with that of inactivating area LIP, which is closely connected to the FEF and is also involved in attention mechanisms. Using exactly the same task, we have shown previously that LIP inactivation causes significant deficits in contralesional target detection during covert visual search (Wardak et al., 2004). There were, however, some notable differences with the effects of FEF inactivation. In particular, we found that LIP inactivation produced deficits, the magnitude of which depended on task difficulty, whereas FEF inactivation produced equally severe deficits in the conjunction search, difficult feature, and easy feature search tasks. Therefore, the effects of FEF inactivation cannot be explained by a mere repercussion of FEF inactivation on the connected area LIP. Another difference is the error rate, 
which was higher after FEF than LIP inactivation mainly because of the high frequency of ipsiversive saccades. This could be a result of an impairment in fixation combined to an imbalance between contraversive and ipsiversive attention orienting tendencies.

What then is the specific contribution of FEF to attention mechanisms, and how does it differ from that of parietal area LIP? We can discuss this in relation with two canonical concepts in the literature on visual search and attention. One is the process of shifting the spatial focus of attention, which serves to enhance locally visual scene analysis (Posner, 1980). The other is the computation of a salience map for visual selection, which results from the competitive interactions between the various elements of the visual scene and from a combination of bottom-up (intrinsic visual features) and topdown (previous knowledge) factors (Desimone and Duncan, 1995; Treue, 2003).

The fact that the deficits caused by FEF inactivation were of the same magnitude in all three visual search conditions, whether the monkeys had to detect a highly salient target object or one that was hidden among very similar objects, argues rather against an important role of the FEF in visual selection. If this was the case, one would have expected to find the FEF, just like LIP, to be more critically involved in conditions in which the target was difficult to individuate than when it was highly salient. This difference between FEF and LIP echoes particularly well what has been described in human functional magnetic resonance imaging (Culham et al., 2001). In this study, the intraparietal and inferior parietal lobule activations increase with attentional load, whereas the FEF activation is present even at the lowest load and is invariant. However, the absence of a saliency effect after FEF inactivation contradicts data from single unit studies showing a relationship between the strength of the visual neuronal activity and target saliency (Bichot et al., 2001). We therefore suggest that the attentional selection of objects in the visual scene might take place in areas upstream from the FEF, and that part of the neuronal modulations exhibited by FEF neurons might be caused by signals received from other areas, such as LIP, rather than computed locally.

Could then the FEF participate in that other aspect of attentional control, namely spatially shifting the focus of attention? Visual search is not a well-suited paradigm to answer this question, because it allows little control over how attention is deployed during performance of the task. Nevertheless, the behavioral strategy and the characteristics of the ipsilesional deficit that we observed in one of the monkeys provide some support for the hypothesis that the FEF contributes to generating contraversive shifts of attention. It could certainly be objected that if this was the main function of the FEF, the visual search impairments we measured should have been proportional to the number of items in the display, because more shifts of attention should be required when there are more objects to inspect. However, the spatial layout of the stimuli in our search arrays was not such that multiple contraversive shifts were systematically needed to detect the target. In the two- and four-item configurations, all objects are located at the same distance of the midline in the contralateral hemifield. Thus, with a single contraversive shift, eventually followed by a vertical shift of attention, the target could be detected. In the eight-item case, monkeys could in principle make multiple

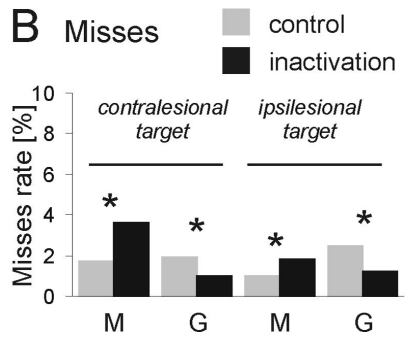

\section{Abortive saccades}

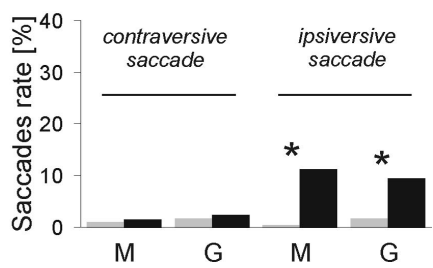

shifts of attention, but if they systematically made a contraversive shift to the most eccentric location, all subsequent shifts would have been directed vertically or ipsilaterally. Also, one needs to keep in mind that more than one object can be processed during a single attentional "fixation." Therefore, it is possible that a mean close to one contraversive shift of attention was sufficient to find the target in all display configurations. The increase in detection time that we observed would therefore mainly reflect a slower initiation of contraversive shifts of attention and/or the cost of redirecting attention if biased ipsilesionally. More adapted tasks should be used in the future to test this hypothesis directly.

The concept of the FEF as an attention shifter raises the issue of the functional relationship between attention shifts and saccadic eye movements within this structure. Our results cannot provide a definitive answer to the question as to whether the two deficits we observed are functionally related. We can only conclude that the FEF is functionally involved in both saccades and attention. Initially, it has been proposed that the two mechanisms were identically coded within the FEF, and that a peripheral inhibition of the eye movements was present in the case of a covert shift of attention ["premotor theory of attention" (Rizzolatti et al., 1987)]. This hypothesis receives support from the results of microstimulation studies (Moore and Fallah, 2001; Moore and Amstrong, 2003), because a microstimulation of the FEF below the saccadic threshold mimics an attentional shift. However, other studies suggest a dissociation or an independence of the two mechanisms (Juan et al., 2004; Thompson et al., 2005), arguing that several cellular populations coexist in the FEF with combinations of motor, visual, and attentional properties (Bruce and Goldberg, 1985; Kodaka et al., 1997) and that it is possible that moving the eyes and moving the attention are the results of two different computations of the input signals and/or involve two different subpopulations with distinct input and output connections.

Functional imaging in humans shows an involvement of the human FEF in both saccadic and attentional mechanisms (Corbetta et al., 1998). The FEF participates in visual search tasks but also in tasks involving discrete shifts of attention (Donner et al., 2000; Beauchamp et al., 2001). Our current findings are therefore consistent with results obtained in humans. Lesions of the frontal cortex that include the FEF produce saccadic deficits as well as attentional deficits and neglect (Heilman and Valenstein, 1972; Rivaud et al., 1994; Rueckert and Grafman, 1996). Two additional parallels can be drawn in relation to the deficits we obtained in the monkey. First, the patients with FEF lesions (with or without accompanying prefrontal cortex lesions) fail to suppress saccades to ipsilesional objects (Guitton et al., 1985; Husain and Kennard, 1997), just as the monkeys we tested in the visual search task. Second, a patient with frontal lobe lesions and neglect is 
described as showing deficits only in the presence of ipsilesional distractors (Husain and Kennard, 1997). This observation also is consistent with our results, because a simple detection task with no distractor objects showed no deficits.

In conclusion, this study shows that the monkey FEF is involved in both saccadic eye movements and visual attention. Its reversible inactivation produces a set of deficits that are very close to those observed in human patients and thus constitutes a valuable model to investigate further the neural mechanisms of selective attention.

\section{References}

Andersen RA, Asanuma C, Essik G, Siegel RM (1990) Cortico-cortical connections of anatomically and physiologically defined subdivisions within the inferior parietal lobule. J Comp Neurol 296:65-113.

Astafiev SV, Shulman GL, Stanley CM, Snyder AZ, Van Essen DC, Corbetta M (2003) Functional organization of human intraparietal and frontal cortex for attending, looking, and pointing. J Neurosci 23:4689-4699.

Beauchamp MS, Petit L, Ellmore TM, Ingeholm J, Haxby JV (2001) A parametric fMRI study of overt and covert shifts of visuospatial attention. NeuroImage 14:310-321.

Bichot NP, Schall JD (1999) Saccade target selection in macaque during feature and conjunction visual search. Vis Neurosci 16:81-89.

Bichot NP, Thompson KG, Chenchal Rao S, Schall JD (2001) Reliability of macaque frontal eye field neurons signaling saccade targets during visual search. J Neurosci 21:713-725.

Bisley JW, Goldberg ME (2003) Neuronal activity in the lateral intraparietal area and spatial attention. Science 299:81-86.

Blatt GJ, Andersen RA, Stoner GR (1990) Visual receptive field organization and cortico-cortical connections of the intraparietal area (area LIP) in the macaque. J Comp Neurol 299:421-445.

Bruce CJ, Goldberg ME (1985) Primate frontal eye fields. I. Single neurons discharging before saccades. J Neurophysiol 53:603-635.

Bruce CJ, Goldberg ME, Bushnell MC, Stanton GB (1985) Primate frontal eye fields. II. Physiological and anatomical correlates of electrically evoked eye movements. J Neurophysiol 54:714-734.

Bullier J, Schall JD, Morel A (1996) Functional streams in occipito-frontal connections in the monkey. Behav Brain Res 76:89-97.

Corbetta M, Akbudak E, Conturo TE, Snyder AZ, Ollinger JM, Drury HA, Linenweber MR, Petersen SE, Raichle ME, Van Essen DC, Shulman GL (1998) A common network of functional areas for attention and eye movements. Neuron 21:761-773.

Culham JC, Cavanagh P, Kanwisher NG (2001) Attention response functions: characterizing brain areas using fMRI activation during parametric variations of attentional load. Neuron 32:737-745.

Desimone R, Duncan J (1995) Neural mechanisms of selective visual attention. Annu Rev Neurosci 18:193-222.

Dias EC, Segraves MA (1999) Muscimol-induced inactivation of monkey frontal eye field: effects on visually and memory-guided saccades. J Neurophysiol 81:2191-2214.

Donner T, Kettermann A, Diesch E, Ostendorf F, Villringer A, Brandt SA (2000) Involvement of the human frontal eye field and multiple parietal areas in covert visual selection during conjunction search. Eur J Neurosci 12:3407-3414.

Goldberg ME, Bushnell MC (1981) Behavioral enhancement of visual responses in monkey cerebral cortex. II. Modulation in frontal eye fields specifically related to saccades. J Neurophysiol 46:773-787.

Guitton D, Buchtel HA, Douglas RM (1985) Frontal lobe lesions in man cause difficulties in suppressing reflexive glances and in generating goaldirected saccades. Exp Brain Res 58:455-472.

Hays AV, Richmond BJ, Optican LM (1982) A UNIX-based multipleprocess system for real-time data acquisition and control. WESCON Conf Proc 2:1-10.

Heilman KM, Valenstein E (1972) Frontal lobe neglect in man. Neurology 22:660-664.

Husain M, Kennard C (1997) Distractor-dependant frontal neglect. Neuropsychologia 35:829-841.

Juan CH, Shorter-Jacobi SM, Schall JD (2004) Dissociation of spatial attention and saccade preparation. Proc Natl Acad Sci USA 101:15541-15544.

Judge SJ, Richmond BJ, Chu FC (1980) Implantation of magnetic search coils for measurements of eye position: an improved method. Vision Res 20:535-538.

Kodaka Y, Mikami A, Kubota K (1997) Neuronal activity in the frontal eye field of the monkey is modulated while attention is focused on to a stimulus in the peripheral visual field, irrespective of eye movement. Neurosci Res 28:291-298.

Malpeli JG (1999) Reversible inactivation of subcortical sites by drug injection. J Neurosci Methods 86:119-128.

Martin JH, Ghez C (1999) Pharmacological inactivation in the analysis of the central control of movement. J Neurosci Methods 86:145-159.

Moore T, Amstrong KM (2003) Selective gating of visual signals by microstimulation of frontal cortex. Nature 421:370-373.

Moore T, Fallah M (2001) Control of eye movements and spatial attention. Proc Natl Acad Sci USA 98:1273-1276.

Moore T, Fallah M (2004) Microstimulation of the frontal eye field and its effect on covert spatial attention. J Neurophysiol 91:152-162.

Posner MI (1980) Orienting of attention. Q J Exp Psychol 32:3-25.

Rivaud S, Müri RM, Gaymard B, Vermersch AI, Pierrot-Deseilligny C (1994) Eye movement disorders after frontal eye field lesions in humans. Exp Brain Res 102:110-120.

Rizzolatti G, Riggio L, Dascola I, Umilta C (1987) Reorienting attention across the horizontal and vertical meridian: evidence in favor of a premotor theory of attention. Neuropsychologia 25:31-40.

Rueckert L, Grafman J (1996) Sustained attention deficits in patients with right frontal lesions. Neuropsychologia 34:953-963.

Schall JD, Hanes DP (1993) Neural basis of saccade target selection in frontal eye field during visual search. Nature 366:467-469.

Schiller PH, Chou I (2000) The effects of anterior arcuate and dorsomedial frontal cortex lesions on visually guided eye movements in the rhesus monkey. I. Single and sequential targets. Vision Res 40:1609-1626.

Sommer MA, Tehovnik EJ (1997) Reversible inactivation of macaque frontal eye field. Exp Brain Res 116:229-249.

Stanton GB, Bruce CJ, Goldberg ME (1995) Topography of projections to posterior cortical areas from the macaque frontal eye fields. J Comp Neurol 353:291-305.

Tehovnik EJ, Sommer MA (1997) Electrically evoked saccades from the dorsomedial frontal cortex and frontal eye fields: a parametric evaluation reveals differences between areas. Exp Brain Res 117:369-378.

Thompson KG, Hanes DP, Bichot NP, Schall JD (1996) Perceptual and motor processing stages identified in the activity of macaque frontal eye field neurons during visual search. J Neurophysiol 76:4040-4055.

Thompson KG, Biscoe KL, Sato TR (2005) Neuronal basis of covert spatial attention in the frontal eye field. J Neurosci 25:9479-9487.

Treue S (2003) Visual attention: the where, what, how and why of saliency. Curr Opin Neurobiol 13:428-432.

Wardak C, Olivier E, Duhamel JR (2004) A deficit in covert attention after parietal cortex inactivation in the monkey. Neuron 42:501-508. 